\title{
Apoptose-Mechanismen in der Onkogenese von Hauttumoren
}

\section{The Regulation of Apoptosis Signalling Pathways During the Development of Skin Tumors}

Autor

Institut

\section{Leverkus}

Klinik für Dermatologie und Venerologie, Otto-von-Guericke-Universität Magdeburg (Direktor: Prof. Dr. Harald Gollnick)

\section{Bibliografie}

Dol 10.1055/s-2008-1077553

Akt Dermatol 2008; 34:

313-318 @ Georg Thieme

Verlag KG Stuttgart · New York ISSN 0340-2541

Korrespondenzadresse Univ.-Prof. Martin Leverkus Klinik für Dermatologie und Venerologie

Otto-von-Guericke-Universität Leipziger Straße 44 39120 Magdeburg martin.leverkus@med.ovgu.de

\section{Zusammenfassung \\ $\nabla$}

Tumorerkrankungen der Haut werden zentral durch die Fähigkeit der Tumorzellen, im Rahmen von Tumorinitiation und -progression Apoptoseresistenz zu erlangen, befördert. So trägt die Apoptoseresistenz entscheidend dazu bei, dass Hauttumorerkrankungen wie das maligne Melanom und das Plattenepithelkarzinom im metastasierten Zustand therapeutisch nur schwer beeinflussbar sind und eine kurative Behandlung dieser Tumorstadien erschwert ist. Ebenso bedingt das multilokuläre Auftreten von Basalzellkarzinomen eine nicht unerhebliche Komorbidität, sodass auch diese Entität neuer Therapiefor-

\section{Einleitung}

$\nabla$

Apoptose, auch programmierter Zelltod genannt, ist ein phylogenetisch hochkonserviertes und zellintrinsisches Selbstmordprogramm, das von entscheidender Bedeutung für die Aufrechterhaltung der Gewebehomöostase ist. Es ist heute klar, dass neben der Fähigkeit zu Angiogenese, Überwindung der Seneszenz und der Möglichkeit zu Gewebeinvasion und Metastasierung weitere zentrale Bedingungen durch die Tumorzelle erfüllt sein müssen, damit sich ein Tumor „erfolgreich“ im Organismus ausbreiten kann: So ist über die Unempfindlichkeit gegenüber antiproliferativen Signalen und der Fähigkeit zur Eigenversorgung mit Wachstumssignalen hinaus - in besonderem Maße die Apoptoseresistenz für die Entstehung von Tumorerkrankungen der Haut bedeutsam [14]. Durch ein „Zuwenig“ an Apoptose kann die Krebsentstehung und Progression von Neoplasien der Haut gefördert werden, während ein „Zuviel“ an Apoptose zur Involution von

\footnotetext{
* Vortrag anlässlich des Jahressymposiums der Berliner Stiftung für Dermatologie am 31.5. 2008.
}

men bedarf. In den letzten Jahren mehrt sich die Evidenz, dass durch gezielte Beeinflussung zentraler Schaltstellen der apoptotischen Signaltransduktionskaskade eine bessere Behandlung dermatoonkologischer Patienten ermöglicht werden könnte. Dazu gehören p53-Reaktivatoren, SonicHedgehog-Antagonisten, aber auch Bcl-2-Antagonisten, IAP-Antagonisten oder TodesrezeptorAgonisten, die sich gegenwärtig in der präklinischen oder frühen klinischen Erprobung befinden. Der Artikel fasst den gegenwärtigen Stand zusammen und gibt einen Ausblick auf potenzielle mechanismusbasierte Therapieoptionen zur Durchbrechung der Apoptoseresistenz bei dermatoonkologischen Erkrankungen.

Hauttumoren führt, wie dies am Beispiel des Keratoakanthoms gezeigt werden kann [4]. Apoptosevorgänge laufen nicht etwa chaotisch $\mathrm{ab}$, sondern stellen einen hochgradig regulierten zellulären Prozess dar, der vermutlich ähnlich komplex wie der Zellzyklus reguliert ist [10]. In der Signaltransduktionskaskade zur Auslösung von Apoptose ist eine Gruppe von Proteasen, sogenannte Caspasen, von zentraler Bedeutung [20]. Eine Untergruppe der Caspasen, die sogenannten Initiator-Caspasen (Caspase-8 und -10) können über einen rezeptorvermittelten Signalweg (extrinsischer Apoptose-Signalweg) die Effektor-Caspasen (Caspase-3, -6, -7) aktivieren (siehe $\odot$ Abb. 1). Im Gegensatz dazu steht der intrinsische Signalweg der Apoptose, der über die Mitochondrien die Apoptose-Kaskade aktiviert [8]. Der rezeptorvermittelte Apoptose-Signalweg wird durch Bindung von TodesrezeptorLiganden an ihre spezifischen Rezeptoren auf der Zelloberfläche aktiviert. Man kennt heute acht verschiedene Todesrezeptoren, nämlich TNF-R1, CD95, TRAIL-R1, TRAIL-R2, DR-3, DR-6, EDAR, sowie den NGF-Rezeptor [22]. Nach Stimulation des Rezeptors mit dem Liganden kommt 


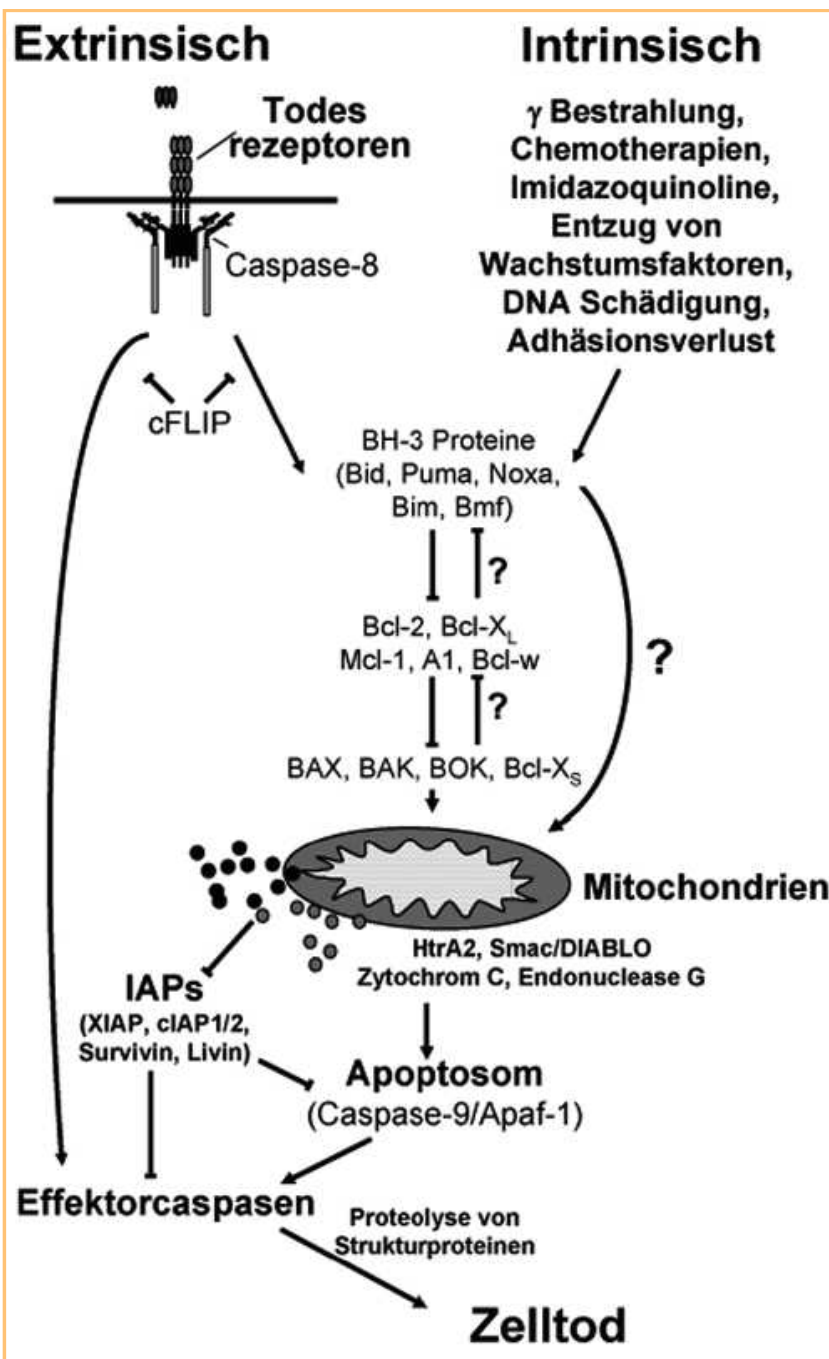

Abb. 1 Die Signaltransduktionskaskade der Apoptose. Schematische Darstellung des derzeitigen Verständnisses der apoptotischen Signaltransduktionskaskade. Es wird ein intrinsischer von einem extrinsischen Apoptose-Signalweg unterschieden, die beide die Aktivierung von Effektorcaspasen initiieren. Effektorcaspasen induzieren durch Proteolyse die Modifikation zahlreicher Substrate der Zelle, die den typischen apoptotischen Phänotyp der Zelle ausbilden (weitere Details siehe auch die ausführliche Darstellung im Text).

es zur Ausbildung einer als „Death-inducing signalling complex“ (DISC) bezeichneten intrazellulären Aktivierungsplattform. Durch die Rekrutierung verschiedener Adaptermoleküle, wie beispielsweise FADD, wird Caspase-8 zum DISC rekrutiert. Dies führt zur autokatalytischen Aktivierung dieser zentralen Initiator-Caspase. Die aktivierte Caspase-8 führt über die Initiierung der nachgeschalteten Caspase-Kaskade schließlich zum Zelltod durch proteolytische Spaltung einer Vielzahl zellulärer Substrate, was anhand der typischen morphologischen und biochemischen Charakteristika nachgewiesen werden kann [20]. Alternativ kann die Aktivierung von Caspasen über den intrinsischen Signalweg der Apoptose ausgelöst werden. Dieser Signalweg kann durch Stressfaktoren, wie DNA-Schädigung, Chemotherapien, Verlust der Adhäsion (sog. Anoikis), aber auch durch den Entzug von Wachstumsfaktoren aktiviert werden. Durch diese Signale werden proapoptotische Moleküle (z.B. Cytochrom-C, HtrA2, Smac/DIABLO, Endonuclease G) aus dem intermembra- nösen Raum der Mitochondrien freigesetzt. Diese Moleküle können einerseits eine weitere hochmolekulare Aktivierungsplattform der Caspase-9 (Apoptosom) aktivieren, andererseits können durch diese aus den Mitochondrien freigesetzten Proteine auch caspaseunabhängige Formen des Zelltodes aktiviert werden [34].

\section{Die Entwicklung von Apoptose-Defekten während der Tumorigenese kutaner Neoplasien $\nabla$}

Die Signaltransduktionskaskade von Apoptose kann in Tumorzellen auf verschiedenen Ebenen gestört sein. So können beispielsweise Proteine der Bcl-2-Familie, die von zentraler Bedeutung für die Regulation des intrinsischen Apoptose-Signalweges verantwortlich sind, aufreguliert werden [9], wie es beispielsweise beim Basalzellkarzinom der Haut bekannt ist [26]. Andererseits kann es zur Blockade dieses Signalweges durch Verlust der sogenannten BH3-Proteine kommen, die als differenzielle Sensoren zellulären Stresses verstanden werden können. Dies konnte für verschiedene Tumorentitäten, u.a. beim malignen Melanom gezeigt werden [30]. Zu weiteren spezifischen Signalmolekülen der Bcl-2-Familie oder anderer Apoptose-Signalmoleküle sind insbesondere zur systematischen Expression in vivo nur wenige Untersuchungen veröffentlicht worden [11].

\section{Apoptoseresistenz beim Plattenepithelkarzinom der Haut \\ $\nabla$}

Von zentraler Bedeutung bei der Entstehung des Plattenepithelkarzinoms der Haut ist das Tumorsuppressorprotein p53. p53-Mutationen und damit die Inaktivierung dieses durch DNASchädigung aktivierten Signalweges können bereits in nur mikroskopisch UV-geschädigter menschlicher Haut gefunden werden, und bei Patienten mit fortgeschrittenem Lichtschaden können großflächig konfluierende Felder p53-mutanter Keratinozyten gefunden werden, die das mikroskopische Korrelat einer Feldkanzerisierung darstellen könnten [5]. In diesem Zusammenhang ist es bedeutsam, dass in photogeschädigter Haut das mutierte, meist nicht mehr zur Transkription von Zielproteinen fähige p53-Protein akkumuliert. In verschiedenen experimentellen Systemen konnte nachgewiesen werden, dass möglicherweise dieses mutierte p53 in der Zelle andere Gene bzw. Transkriptome aktivieren kann, als es p53 in seiner wildtypischen Variante kann („gain of function“-Mutation) [38]. Die zentrale Bedeutung des Verlustes der Wildtyp-Funktion p53 konnten auch elegante Mausmodelle herausstellen, die zeigten, dass durch den Verlust der Funktion von Wildtyp-p53 die onkogeninduzierte Seneszenz verhindert und gleichzeitig die Apoptoseresistenz in fortgeschrittenen Tumorstadien aufrecht erhalten wird $[36,41]$. Damit ist heute klar, dass der p53-Verlust nicht nur in frühen Tumorstadien des Plattenepithelkarzinoms der Haut, sondern durch die gesamte Tumorprogression aufrechterhalten werden muss und somit ein potenzielles therapeutisches Ziel darstellen könnte. 


\section{Molekulare Mechanismen der Tumorigenese des Basalzellkarzinoms \\ $\nabla$}

Der häufigste menschliche Tumor, das Basalzellkarzinom, war über sehr lange Zeit experimentell nur schwer zugänglich, da die Tumoren weder im Mausmodell induzierbar waren, noch humane Tumoren zufriedenstellend in Zellkultur überführt werden konnten [2]. Arbeiten der letzten Dekade haben nunmehr überzeugend darstellen können, dass es beim Basalzellkarzinom zur dauerhaften Aktivierung des Sonic-Hedgehog-Signalwegs kommt. Dieser eigentlich embryonal bedeutsame Signalweg fördert bei Basalzellkarzinomen das Tumorwachstum [15]. Ein zentrales Suppressor-Protein des Sonic-Hedgehog-Signalweges, Patched (PTCH), ist beim Basalzellnaevus-Syndrom (GorlinGoltz-Syndrom) heterozygot mutiert [18], und eine heterozygote Deletion dieses Gens in der Maus erlaubt Untersuchungen zur UV- oder $\gamma$-bestrahlungsinduzierten Entstehung von Basalzellkarzinomen [1]. Auf dieser wegweisenden Erkenntnis aufbauende Arbeiten verschiedener Arbeitsgruppen konnten zeigen, dass alternativ das durch Patched inaktivierte Signalprotein Smoothened (SMO), das durch Patched in der KeratinozytenMembran inaktiviert wird, aktivierende Mutationen bei sporadischen Basalzellkarzinomen tragen kann [16]. So gilt heute zusammenfassend als sicher, dass ein konstitutiv aktiver SonicHedgehog-Signalweg, der zur Aktivierung von Transkriptionsfaktoren der Familie der Gli-Proteine führt [27], beim BCC von entscheidender Bedeutung ist [2]. Gli-Proteine aktivieren wiederum nicht nur eine autokrine EGF-Stimulation [3], sondern regeln auch zentrale antiapoptotische Moleküle wie cFLIP auf, was für die Apoptoseresistenz von BCC bedeutsam ist [19].

\section{Apoptoseresistenz beim malignen Melanom \\ $\nabla$}

Beim aggressivsten Tumor der Haut, dem malignen Melanom, das aus primär neuroektodermalen Melanozyten oder Vorläuferzellen hervorgeht, konnten auf verschiedenen Ebenen Apoptoseresistenz-Mechanismen identifiziert werden, die zur hochgradigen Therapieresistenz gegenüber herkömmlichen Chemo- und $\gamma$-Bestrahlungstherapien der metastasierten Tumorstadien beitragen. So wurde beispielsweise ein kritischer Inhibitor der Todesrezeptor-vermittelten Apoptose, cFLIP, als in Melanomzellen hochexprimiertes Molekül identifiziert [17]. Weiterhin konnten Korrelationen zur Expression verschiedener Bcl-2-Familienmitglieder dargestellt werden, die mit der Prognose des malignen Melanoms korrelierten [11]. Verschiedene, beim malignen Melanom kritische Signalwege (p16 ${ }^{\text {INK4A }}$, p14ARF, die zyklinabhängige Kinase 4 [CDK4]), aber auch N-Ras, B-Raf, sowie Mitogen-aktivierte Protein-Kinase-(MAPK)-regulierte Signale fördern nicht nur die Proliferation, sondern auch die Seneszenz der Tumorzellen, sodass insgesamt bei malignen Melanomen proliferative Signalwege aktiviert sind, die jedoch zur malignen Tumorentstehung nicht hinreichend sind [13]. Diese Signalwege blockieren andererseits durch Induktion antiapoptotischer Moleküle der Bcl-2-Familie oder Inaktivierung von BH3-Molekülen wie Bad die apoptotische Signalkaskade [24], sodass beim malignen Melanom ebenso das Wechselspiel proliferativer und apoptotischer Signalwege bedeutsam ist.

\section{Mechanismusbasierte Therapieoptionen durch Induktion von Apoptose bei Hauttumoren \\ $\checkmark$}

Obwohl der Anteil metastasierungsfähiger Plattenepithelkarzinome der Haut insgesamt gering ist, sind im Falle der Metastasierung die herkömmlichen chemotherapeutischen Verfahren nicht ausreichend wirksam. Ebenso sind die Möglichkeiten zur aggressiven und potenziell nebenwirkungsreichen Polychemotherapie zumeist begrenzt, da aufgrund der hohen Inzidenz im fortgeschrittenen Lebensalter es sich weitgehend um Patienten mit erheblichen Komorbiditäten handelt [21]. Die Induktion von Apoptose bei dieser Tumorentität stellt daher einen innovativen Therapieansatz dar, der die Prognose im Stadium III oder IV dieser Tumorentität verbessern könnte [6]. Durch die obligate Mutation von p53 und der Akkumulation des mutanten Proteins fehlt die transkriptionelle Aufregulation proapoptotischer Moleküle von BAX bzw. von anderen proapoptotisch wirksamen BH3-Proteinen (wie z.B. Puma und Noxa), die an der Auslösung p53-abhängiger Apoptose beteiligt sind. So ist es nicht verwunderlich, dass die Apoptoseinduktion durch Chemotherapien oder auch Bestrahlungstherapien unterdrückt ist und daher nur unzureichend wirksam sein kann. Aufgrund der Anreicherung des mutanten p53 in den Tumorzellen des Plattenepithelkarzinoms könnte jedoch eine Apoptoseinduktion durch Reaktivierung von p53 eine innovative Therapiemodalität darstellen $[28,39]$. So wurden von verschiedenen Arbeitsguppen Moleküle identifiziert, die in der Lage sind, die mutanten Formen von p53 in die Wildtypkonfiguration zu überführen. In präklinischen Modellen konnte erfolgreich mutantes p53 reaktiviert werden und so p53-abhängige Apoptose in Tumorzellen induziert werden [7]. Ebenso konnte vor Kurzem erfolgreich von der Unterdrückung von UV-induzierten Tumoren in einem Mausmodell durch Anwendung des p53-Reaktivators CP31398 berichtet werden [32]. Damit könnte die Reaktivierung von p53 einerseits eine erfolgversprechende Therapie zur Vermeidung der Progression einer Feldkanzerose zu invasiven Tumoren sein, andererseits eine interessante Option bei inoperablem oder metastasiertem Plattenepithelkarzinom der Haut darstellen ( $\bullet$ Abb. 2, TZ 1).

Ausgehend von der Erkenntnissen, dass der Sonic Hedghog-Signalweg beim Basalzellkarzinom der Haut konstitutiv aktiviert ist, wurden kleinmolekulare Substanzen entwickelt, die spezifisch den Sonic-Hedgehog-Signalweg blockieren können. Als Leitsubstanz konnte das Cyclopamin beschrieben werden [31]. Aufbauende Substanzen, wie das CUR61414, Purmorphamin oder GDC-0449, die sich in der präklinischen bzw. frühen klinischen Prüfung befinden, werden gegenwärtig für die klinische Anwendung entwickelt [29]. Interessanterweise konnte Cyclopamin auch in einem Mausmodell das UV-vermittelte Auftreten von Basalzellkarzinomen in einem therapeutisch relevanten experimentellen Ansatz hemmen. So konnte durch Gabe des Antagonisten die rasche Induktion von Apoptose in den Tumorzellen festgestellt werden, die über die endogene Aktivierung des Todesrezeptors CD95 vermittelt zu sein scheint [2] (siehe - Abb. 2; TZ 2). Weiterhin konnte mit einem oral verfügbaren Sonic-Hedgehog-Antagonisten (GDC-0449) vor kurzem erfolgreich eine Phase-I-Studie beim fortgeschrittenen, multifokalen oder metastasierenden Basalzellkarzinom mit ermutigendem Ergebnis abgeschlossen werden [37]. So konnte eine klinische Regression bei 8 von 9 behandelten Patienten per Apoptoseinduktion nachgewiesen werden. Schon die nahe Zukunft lässt also hoffen, dass diese Substanzen Eingang in das therapeutische Arsenal des onkologisch tätigen Dermatologen finden werden. 


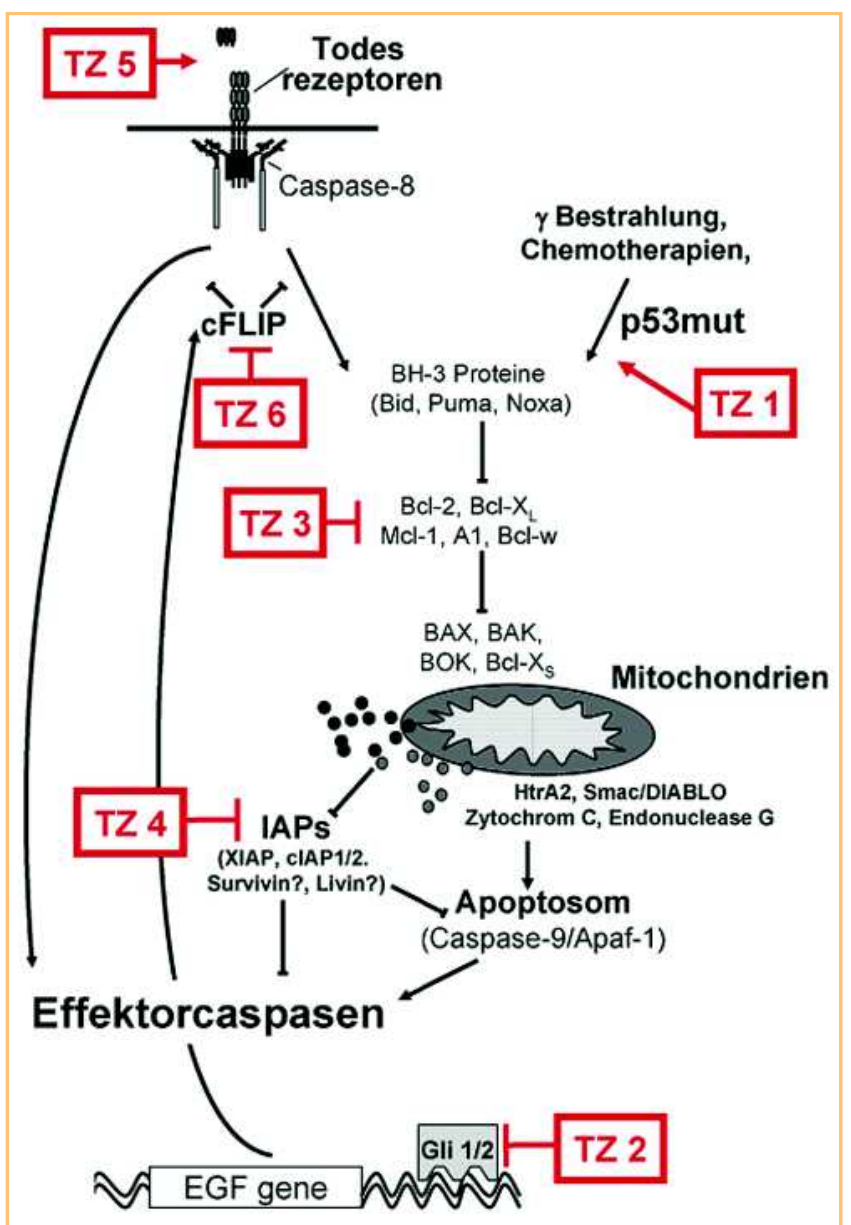

Abb. 2 Therapeutische potenzielle Ansatzmöglichkeiten zur Durchbrechung der Apoptoseresistenz. Dargestellt sind potenzielle Zielmoleküle, die direkt oder indirekt in die apoptotische Signaltransduktionskaskade eingreifen. Einzelheiten der Aktivierung von Zielmolekülen sind im Text jeweils mit dem Kürzel „Therapeutisches Ziel“ (TZ 1-6) gekennzeichnet (Einzelheiten siehe auch die ausführliche Darstellung im Text).

\section{Generelle Induktoren von Apoptose-Signalwegen $\nabla$}

Die aufgeführten Therapieoptionen zu spezifischen Signalwegen und einzelnen Tumorentitäten stellen nur eine erste, gleichwohl Tumorentitäts-spezifische Form der Therapie dar, die zur Behandlung von Hauttumoren eingesetzt werden könnte. Darüber hinaus sind weitere Substanzen, deren Entwicklung direkt auf dem Verständnis von Apoptose-Signalwegen beruht, potenziell zur Therapie von Tumorerkrankungen einsetzbar. Ausgehend von den Befunden zur kritischen Regulation des mitochondrialen Signalweges der Apoptose durch Bcl-2-Familienmitglieder konnten in den letzten Jahren Substanzen entwickelt werden, die in der Lage sind, antiapoptotische BCL-2-Familienmitglieder zu blockieren. So konnte mit ABT-737 [25] erstmals eine spezifisch apoptoseinduzierende Substanz identifiziert werden, die direkt den mitochondrialen Signalweg der Apoptose aktiviert. Vor Kurzem konnte mit der oral verfügbaren Substanz ABT-263 ein auf ABT-737 basierendes Derivat beschrieben werden (○ Abb. 2, TZ 3) [33]. Auch beim Plattenepithelkarzinom der Haut scheint eine selektive Wirksamkeit dieser Substanz auf Tumorzellen vorzuliegen, während primäre humane Keratinozyten in vitro resistent gegenüber dem apoptoseinduzierenden Signal durch ABT-737 sind (P. Geserick und M. Leverkus, unveröffent- lichte Beobachtung). Ob diese Substanzen als Monotherapien oder in Kombinationen mit beispielsweise Chemotherapeutika erfolgversprechend zum Einsatz gebracht werden können, bedarf zukünftig weiterer experimenteller und präklinischer Studien.

„Inhibitor of apoptosis“-Proteine (IAPs) sind eine Gruppe von Eiweißen, die die Apoptose in der Effektorphase hemmen können. Diese Moleküle vermitteln insbesondere die Aktivierung von Effektorcaspasen. Zu den IAP-Proteinen gehören acht Moleküle, und zwar XIAP, cIAP1, cIAP2, Survivin, NAIP, ILP-2, BRUCE (Apollon) und Livin (ML-IAP) [35]. Während die antiapoptotische Wirkung zahlreicher IAP-Moleküle noch unklar ist, erreicht XIAP seine antiapoptotische Wirkung durch Blockade der aktivierten Caspase-3, -7 und -9. Weiterhin kann XIAP die Blockade der Aktivierung von Caspase-9 erreichen. IAPs sind ein wichtiger Resistenzfaktor in verschiedenen malignen Tumoren, da sie die apoptotische Signalkaskade an zentraler Schaltstelle blockieren. Es ist nicht verwunderlich, dass sie in vielen Tumorformen vermehrt exprimiert werden und einen weiteren wichtigen Resistenzfaktor gegenüber konventionellen Chemotherapien darstellen. Mit verschiedenen Strategien sind in den letzten Jahren Substanzen entwickelt worden, die direkt die Funktion von XIAP blockieren können, darunter Antisense-Oligonukleotide sowie niedermolekulare Inhibitoren. In präklinischen Studien konnte gezeigt werden, dass kleinmolekulare Smac-Peptide oder Inhibitoren, die die Bindung des aus den Mitochondrien freigesetzten Smac an XIAP vermitteln und so dieses Molekül inaktivieren können, nachfolgend eine Aktivierung von Caspasen in Tumorzellen erreichen. So konnte in verschiedenen Tumorzellen mit diesen Inhibitoren direkt Apoptose induziert werden oder für andere Apoptose-Signale wie beispielsweise Chemotherapeutika sensitiviert werden. Da beispielsweise beim malignen Melanom das IAP-Protein Livin deutlich überexprimiert wird sowie bei Plattenepithelkarzinomen der Haut beispielsweise XIAP in Untergruppen der Tumoren hochexprimiert wird (Peter Geserick und Martin Leverkus, unveröffentlichte Beobachtung), ist davon auszugehen, dass auch IAP-Antagonisten erfolgversprechende Therapeutika zur Behandlung von Hauttumoren sein könnten (siehe - Abb. 2, TZ 4).

Durch Aktivierung von Todesrezeptoren können direkt die zentralen Effektormoleküle der Apoptose, die Effektor-Caspasen-3, -6 und -7 aktiviert werden. Somit stellen Todesliganden bzw. Todesrezeptor-Agonisten eine potenzielle interessante Therapieoption von Tumorerkrankungen dar (vgl. @ Abb. 2, TZ 5). Insbesondere beim Plattenepithelkarzinom der Haut zeigt sich durch differenzielle Expression eines zentralen Inhibitors, cFLIP, eine selektive Sensitivität transformierter Keratinozyten, während fortgeschrittene Tumorstadien cFLIP wiederum aufregulieren (Peter Geserick and Martin Leverkus, unveröffentlichte Beobachtung) [23]. Im Gegensatz zu cFLIP sind in vitro die bislang untersuchten Todesrezeptoren (CD95, TRAIL-Rezeptor [TRAIL-R]-1 sowie TRAIL-R2) homogen auf der Zelloberfläche exprimiert. Damit könnten Aktivatoren des extrinsischen Signalweges der Apoptose auch erfolgversprechende Therapeutika zur Behandlung des Plattenepithelkarzinoms der Haut darstellen. Hierzu werden jedoch weitere präklinische Untersuchungen zur genauen zelltypspezifischen und epidemiologischen Untersuchung an größeren Kollektiven von Primärtumoren und Metastasen erforderlich sein.

Vermutlich durch die hohe Expression des intrazellulären Inhibitors cFLIP zeigen sich viele Melanomzellen resistent gegenüber einem Todesrezeptor-vermittelten Apoptose-Signal. Der in- 
trazelluläre Inhibitor cFLIP wird in seiner Expression auf verschiedenen Ebenen gesteuert und ist aufgrund seiner intrazellulär kurzen Halbwertszeit ein attraktives therapeutisches Ziel (๑ Abb. 2, TZ 6) [22]. Um zu überprüfen, ob Melanomzellen durch cFLIP kritisch gegenüber Todesrezeptor-vermittelten Signalen geschützt sind, konnte mit „knock down“-Experimenten nachgewiesen werden, dass die isolierte Herunterregulation von cFLIP ausreichend ist, um Melanomzellen dramatisch für ein proapoptotisches Signal über Todesrezeptoren zu sensitivieren [12]. Diese Experimente stellen unter Beweis, dass eine Herunterregulation von Inhibitoren der Apoptose ein erfolgversprechendes Konzept zur Tumortherapie sein könnte. Durch verschiedene posttranskriptionelle Mechanismen, die cFLIP regulieren, könnte auch pharmakologisch Zugang zur Aktivierbarkeit dieses Signalweges gefunden werden. Die Translation im Besonderen von cFLIPs wird durch "mammalian target of rapamycin“ (mTOR) reguliert, sodass mTOR-Inhibitoren erfolgversprechend zur Herunterregulation von cFLIP eingesetzt werden könnten. Da cFLIP über NF-kB aufreguliert wird, könnten auch Proteasomen-Inhibitoren zur Herunterregulation von cFLIP verwendet werden. Es wurden zahlreiche weitere pharmakologische Substanzen identifiziert, die gegenüber Todesrezeptor-vermittelter Apoptose sensitivieren können, u.a. 4-(4-chloro-2-methlyphenoxy)-N-Hydroxybutanamide, auch als 5809354 bekannt, das synthetische Tripertenoid 2-Cyano-3,12-Dioxoolean-1,9-bien28-oic-Säure (CDDO) oder Histon-Deazethylase-Hemmer wie beispielsweise die Substanz Suberoylanilid-Hydroxamin-Säure (SAHA). Mehr spezifisch agierende Therapieansätze, wie der Einsatz von siRNAs gegen cFLIP oder aufbauende Verfahren [40] könnten zukünftig ebenfalls zur Therapie des malignen Melanoms erfolgversprechend sein.

\section{Apoptoseresistenz: Problem und Chance bei der Tumortherapie von Hauttumoren? \\ $\nabla$}

In den letzten zwei Dekaden ist das Wissen über tumorspezifisch aktivierte bzw. inaktivierte Signalwege auch bei Tumoren der Haut dramatisch angewachsen. Wir werden gegenwärtig Zeuge einer bemerkenswert breiten Entwicklung von spezifischen Substanzen, die mit hoher Selektivität einzelne Signalwege von Tumorzellen beeinflussen können. Der rationelle Einsatz bei Tumorerkrankungen der Haut bedarf daher weiterer, möglicherweise individualisierter systembiologischer Ansätze, um die ideale, nebenwirkungsarme und somit „maßgeschneiderte“ Therapie für fortgeschrittene Melanom- oder Plattenepithelkarzinom-Patienten zu entwickeln. Neben den gegenwärtig entwickelten Inhibitoren von proliferativen Signalwegen werden dabei Modulatoren von Apoptose-Signalwegen mit großer Sicherheit eine wichtige Rolle spielen.

\section{Förderung}

Die Forschungsarbeiten von M. L. werden durch die Deutsche Krebshilfe (106849), die Stiftung Deutsches Hautforschungszentrum sowie die Deutsche Forschungsgemeinschaft (Le 953/5-1) unterstützt.

\section{Abstract}

\section{The Regulation of Apoptosis Signalling Pathways During the Development of Skin Tumors $\nabla$}

The prognosis of cutaneous neoplasia such as squamous cell carcinoma (SCC), basal cell carcinoma (BCC), or malignant melanoma (MM) are severely influenced by the ability of tumor cells to develop or maintain apoptosis resistance throughout tumor progression and metastasis. Furthermore, the ability to overcome apoptosis resistance is a critical factor that determines therapeutic failure or success in metastatic SCC or MM. Moreover apoptosis resistence is a major obstacle to curative efforts in these dermatooncologic diseases. In BCC, the multitude of tumors results in a substantial comorbidity that require innovative treatment options. Over the past years, there is increasing evidence that targeted therapy aiming at the apoptotic signalling cascade in a tumor type-specific manner may result in a better success of oncological treatment. In this context, reactivators of p53, antagonists of sonic hedgehog, but also Bcl-2- or IAP antagonists as well as death receptor agonists that are currently in preclinical or early clinical development may prove to be useful in dermatooncology. The article explains the current knowledge in this rapidly moving field and summarizes potential mechanism-based therapeutic options to overcome apoptosis resistance in the different forms of skin cancer.

\section{Literatur}

1 Aszterbaum M, Epstein J, Oro A et al. Ultraviolet and ionizing radiation enhance the growth of BCCs and trichoblastomas in patched heterozygous knockout mice. Nat Med 1999; 5: 1285 - 1291

2 Athar M, Tang X, Lee JL et al. Hedgehog signalling in skin development and cancer. Exp Dermatol 2006; 15: 667-677

3 Bigelow RL, Jen EY, Delehedde $M$ et al. Sonic hedgehog induces epidermal growth factor dependent matrix infiltration in HaCaT keratinocytes. J Invest Dermatol 2005; 124: 457 - 465

4 Boukamp P. UV-induced skin cancer: similarities - variations. J Dtsch Dermatol Ges 2005; 3: $493-503$

5 Braakhuis BJ, Leemans CR, Brakenhoff RH. Expanding fields of genetically altered cells in head and neck squamous carcinogenesis. Semin Cancer Biol 2005; 15: 113 - 120

6 Brantsch KD, Meisner C, Schonfisch B et al. Analysis of risk factors determining prognosis of cutaneous squamous-cell carcinoma: a prospective study. Lancet Oncol 2008; 9: $713-720$

7 Bykov VJ, Issaeva N, Shilov A et al. Restoration of the tumor suppressor function to mutant p53 by a low-molecular-weight compound. Nat Med 2002; 8: 282 - 288

8 Chalah A, Khosravi-Far R. The mitochondrial death pathway. Adv Exp Med Biol 2008; 615: 25-45

9 Daniel PT, Schulze-Osthoff K, Belka C, Guner D. Guardians of cell death: the Bcl-2 family proteins. Essays Biochem 2003; 39: 73-88

10 Degterev A, Yuan J. Expansion and evolution of cell death programmes. Nat Rev Mol Cell Biol 2008; 9: 378-390

11 Fecker LF, Geilen CC, Tchernev G et al. Loss of proapoptotic Bcl-2-related multidomain proteins in primary melanomas is associated with poor prognosis. J Invest Dermatol 2006; 126: 1366 - 1371

12 Geserick P, Drewniok C, Hupe $M$ et al. Suppression of CFLIP is sufficient to sensitize human melanoma cells to TRAIL- and CD95L-mediated apoptosis. Oncogene 2008; 27: 3211 - 3220

13 Green CL, Khavari PA. Targets for molecular therapy of skin cancer. Semin Cancer Biol 2004; 14: 63 -69

14 Hanahan D, Weinberg RA. The hallmarks of cancer. Cell 2000; 100: 57 70

15 Huntzicker EG, Estay IS, Zhen $H$ et al. Dual degradation signals control Gli protein stability and tumor formation. Genes Dev 2006; 20: 276 281 
16 Ikram MS, Neill GW, Regl G et al. GLI2 is expressed in normal human epidermis and BCC and induces GLI1 expression by binding to its promoter. J Invest Dermatol 2004; 122: 1503 - 1509

17 Irmler $M$, Thome $M$, Hahne $M$ et al. Inhibition of death receptor signals by cellular FLIP. Nature 1997; 388: 190-195

18 Johnson RL, Rothman AL, Xie J et al. Human homolog of patched, a candidate gene for the basal cell nevus syndrome. Science 1996; 272: $1668-1671$

19 Kump E, Ji J, Wernli M, Hausermann P, Erb P. Gli2 upregulates cFlip and renders basal cell carcinoma cells resistant to death ligand-mediated apoptosis. Oncogene 2008; 27: 3856-3864

20 Lavrik IN, Golks A, Krammer PH. Caspases: pharmacological manipulation of cell death. J Clin Invest 2005; 115: 2665-2672

21 Leiter $U$, Garbe $C$. Epidemiology of melanoma and nonmelanoma skin cancer - the role of sunlight. Adv Exp Med Biol 2008; 624: 89-103

22 Leverkus M, Diessenbacher P, Geserick P. FLIPing the coin? Death receptor-mediated signals during skin tumorigenesis. Exp Dermatol 2008; 17: $614-622$

23 Leverkus $M$, Neumann $M$, Mengling $T$ et al. Regulation of tumor necrosis factor-related apoptosis-inducing ligand sensitivity in primary and transformed human keratinocytes. Cancer Res 2000; 60: 553-559

24 Meier F, Busch S, Lasithiotakis Ket al. Combined targeting of MAPK and AKT signalling pathways is a promising strategy for melanoma treatment. Br J Dermatol 2007; 156: 1204- 1213

25 Oltersdorf T, Elmore SW, Shoemaker AR et al. An inhibitor of Bcl-2 family proteins induces regression of solid tumours. Nature 2005; 435: 677-681

26 Regl G, Kasper M, Schnidar H et al. Activation of the BCL2 promoter in response to Hedgehog/GLI signal transduction is predominantly mediated by GLI2. Cancer Res 2004; 64: 7724-7731

27 Regl G, Neill GW, Eichberger T et al. Human GLI2 and GLI1 are part of a positive feedback mechanism in basal cell carcinoma. Oncogene 2002; 21: 5529-5539

28 Selivanova G, Wiman KG. Reactivation of mutant p53: molecular mechanisms and therapeutic potential. Oncogene 2007; 26: $2243-$ 2254
29 Sinha S, Chen JK. Purmorphamine activates the Hedgehog pathway by targeting smoothened. Nat Chem Biol 2006; 2: 29-30

30 Soengas MS, Lowe SW. Apoptosis and melanoma chemoresistance. Oncogene 2003; 22: 138 - 3151

31 Taipale J, Chen JK, Cooper MK et al. Effects of oncogenic mutations in smoothened and patched can be reversed by cyclopamine. Nature 2000; 406: 1005-1009

32 Tang X, Zhu Y, Han L et al. CP-31398 restores mutant p53 tumor suppressor function and inhibits UVB-induced skin carcinogenesis in mice. J Clin Invest 2007; 117: 3753 - 3764

33 Tse C, Shoemaker AR, Adickes J et al. ABT-263: a potent and orally bioavailable Bcl-2 family inhibitor. Cancer Res 2008; 68: 3421 - 3428

34 van Loo G, Saelens X, van Gurp $M$ et al. The role of mitochondrial factors in apoptosis: a Russian roulette with more than one bullet. Cell Death Differ 2002; 9: 1031 - 1042

35 Vaux DL, Silke J. IAPs, RINGs and ubiquitylation. Nat Rev Mol Cell Biol 2005; 6: 287-297

36 Ventura A, Kirsch DG, McLaughlin ME et al. Restoration of p53 function leads to tumour regression in vivo. Nature 2007; 445: 661 - 665

37 von Hoff DD, Rudin CM, LoRusso PM et al. Efficacy data of GDC-0449, a systemic Hedgehog pathway antagonist, in a first-in-human, first-inclass Phase I study with locally advanced, multifocal or metastatic basal cell carcinoma patients. AACR 2008; Abstract LBG-138

38 Weisz L, Damalas A, Liontos $M$ et al. Mutant p53 enhances nuclear factor kappaB activation by tumor necrosis factor alpha in cancer cells. Cancer Res 2007; 67: 2396-2401

39 Wiman KG. Restoration of wild-type 553 function in human tumors: strategies for efficient cancer therapy. Adv Cancer Res 2007; 97: $321-338$

40 Wolfrum C, Shi S, Jayaprakash KN et al. Mechanisms and optimization of in vivo delivery of lipophilic siRNAs. Nat Biotechnol 2007; 25: $1149-1157$

41 Xue W, Zender L, Miething $C$ et al. Senescence and tumour clearance is triggered by $\mathrm{p} 53$ restoration in murine liver carcinomas. Nature 2007; 445: $656-660$ 